\title{
ON WEAKLY STABLE YANG-MILLS FIELDS OVER POSITIVELY PINCHED MANIFOLDS AND CERTAIN SYMMETRIC SPACES
}

\author{
By Yoshiniro OHnita and Pan Yanglian (Y.L. Pan)
}

\begin{abstract}
In this paper it is proved that for $n \geq 5$ there exists a constant $\delta(n)$ with $1 / 4<\delta(n)<1$ such that any weakly stable Yang-Mills connection over a simple connected compact Riemannian manifold $M$ of dimension $n$ with $\delta(n)$-pinched sectional curvatures is always flat. The pinching constants are possible to compute by elementary functions. Moreover we give some remarks on stability of Yang-Mills connections over certain symmetric spaces.
\end{abstract}

\section{Introduction.}

Let $M$ be an $n$-dimensional compact Riemannian manifold with a metric $g$ and $G$ be a compact Lie group with the Lie algebra $g$. Let $E$ be a Riemannian vector bundle over $M$ with structure group $G$, and let $\mathcal{C}_{E}$ denote the space of $G$-connections on $E$, which is an affine space modeled on the vector space $\Omega^{1}\left(g_{E}\right)$ of smooth 1-forms with values in the adjoint bundle $g_{E}$ of $E$. The Yang-Mills functional of $\mathscr{M}: \mathcal{C}_{E} \rightarrow \boldsymbol{R}$ is

$$
y \mathscr{M}(\nabla)=\frac{1}{2} \int_{M}\left\|F^{\nabla}\right\|^{2} d v o l,
$$

for each $\nabla \in \mathcal{C}_{E}$, where $F^{\nabla}$ is the curvature form of the connection $\nabla$. Note that $F \nabla$ is a smooth section of $\Omega^{2}\left(g_{E}\right)$. The Yang-Mills connection $\nabla \in \mathcal{C}_{E}$ is a critical point of $y \mathscr{M}$. A Yang-Mills connection $\nabla$ is called weakly stable if, for each $\nabla^{t} \in \mathcal{C}_{E}$ with $\nabla=\nabla^{0}$,

$$
\left(d^{2} / d t^{2}\right) \text { g }\left.\mathscr{M}\left(\nabla^{t}\right)\right|_{t=0} \geqq 0 .
$$

$M$ is called Yang-Mills unstable (cf. [K-O-T]) if, for every vector bundle $(E, G)$ over $M$, any weakly stable Yang-Mills connection on $E$ is always fiat. First Simons proved that the Euclidean $n$-sphere $S^{n}$ for $n \geqq 5$ is Yang-Mills unstable ([B-L]). Ever since several persons have investigated the instability of YangMills fields over various Riemannian manifolds; convex hypersurfaces, submani-

Received January 12, 1990. 
folds, compact symmetric spaces (cf. [Ka], [K-O-T], [Pal], [Sh], [Ta], [We]). In [K-O-T] it was shown that the Cayley projective plane $P_{2}$ (Cay) and the compact symmetric space of exceptional type $E_{6} / F_{4}$ are Yang-Mills unstable.

In this paper we first establish the instability theorem for Yang-Mills fields over a simply connected compact Riemannian manifold with sufficiently pinched sectional curvatures. Okayasu [Ok] used the construction and results of Ruh, Grove and Karcher ([Ru], [G-K-R1], [G-K-R2]) to show the instability of harmonic maps into a Riemannian manifold with sufficiently pinched sectional curvatures. By using the same idea, the second named author [Pa2] showed an instability theorem for harmonic maps from a Riemannian manifold with sufficiently pinched sectional curvatures to an arbitrary Riemannian manifold. We will also use it. Next we shall prove some results on weakly stable Yang-Mills fields over certain symmetric spaces. Some of them were stated in [K-O-T] without proof. They supplement results of Laquer [La] which determined the stablity of canonical connections over simply connected compact irreducible spaces. Moreover we prove that a weakly stable Yang-Mills field satisfying a certain condition over a quaternionic projective space $P_{m}(\boldsymbol{H})$ is a $B_{2}$-connection in a sense of [Ni], or equivalently a self-dual connection in a sense of [C-S], and hence it minimizes the Yang-Mills functional.

\section{Preliminaries on Yang-Mills fields.}

Let $\nabla \in \mathcal{C}_{E}$. For any $B \in \Omega^{1}\left(g_{E}\right)$, set $\nabla^{t}=\nabla+t B \in \mathcal{C}_{E}$. The second variational formula formula for the Yang-Mills functional is given as follows ([B-L]);

$$
\begin{aligned}
\left.\left(d^{2} / d t^{2}\right) y \mathscr{M}\left(\nabla^{t}\right)\right|_{t=0} & =\mathfrak{T}^{\nabla}(B, B) \\
& =\int_{M}\left(\mathcal{S}_{0}^{\nabla}(B), B\right) d v o l \\
& =\int_{M}\left\{\left(\mathcal{S}^{\nabla}(B), B\right)-\left(\delta^{\nabla} B, \delta^{\nabla} B\right)\right\} d v o l,
\end{aligned}
$$

where $\mathcal{S}_{0}^{\nabla}(B)=\delta^{\nabla} d^{\nabla} B+\mathcal{F}^{\nabla}(B)$ and $\mathcal{S}^{\nabla}(B)=\Delta \nabla(B)+\mathscr{T}^{\nabla}(B)$. Here $d^{\nabla}$ and $\delta^{\nabla}$ denote the exterior covariant differentiation induced by the connection $\nabla \in \mathcal{C}_{E}$ and its adjoint differential operator, and $\mathscr{T}^{\nabla}$ is a symmetric bundle endomorphism of $T^{*} M \otimes g_{E}$ defined by $\left(\Phi^{\nabla}(b)\right)(X)=\sum_{\imath=1}^{n}\left[F^{\nabla}\left(e_{\imath}, X\right), b\left(e_{\imath}\right)\right]$ for $b \in T{ }_{x}^{*} M \otimes\left(g_{E}\right)_{x}$ and $X \in T_{x} M$, where $\left\{e_{2}\right\}$ is an orthonormal basis of $T_{x} M$.

Let $\left\{\omega^{i}\right\}$ be the dual frame of a local orthonormal frame field $\left\{e_{\imath}\right\}$ in $M$, Throughout this paper we use the summation convention. Set $B=B_{i} \omega^{2}$ and $F^{\nabla}=(1 / 2) F_{\imath j} \omega^{\imath} \wedge \omega^{\jmath}$. Then we have

$$
\begin{aligned}
& d^{\nabla} B=\left(\nabla_{\imath} B_{j}-\nabla_{j} B_{\imath}\right) \omega^{\imath} \wedge \omega^{\jmath}, \\
& \delta^{\nabla} d^{\nabla} B=\left(\nabla_{j} \nabla_{\imath} B_{j}-\nabla_{j} \nabla_{\imath} B_{\imath}\right) \omega^{\imath}, \\
& \mathscr{F}^{\nabla}(B)=\left[F_{\imath \jmath}, B_{\imath}\right] \omega^{\jmath},
\end{aligned}
$$


And (1.1) becomes

$$
\left\|F^{\nabla}\right\|^{2}=\left(F_{\imath \jmath}, F_{\imath j}\right) / 2 \text {. }
$$

$$
\begin{aligned}
& \left.\left(d^{2} / d t^{2}\right) \mathscr{g} \mathscr{M}\left(\nabla^{t}\right)\right|_{t=0} \\
& =\int_{M}\left\{\left(\nabla_{j} \nabla_{\imath} B_{\jmath}, B_{\imath}\right)-\left(\nabla_{j} \nabla_{j} B_{\imath}, B_{\imath}\right)+\left(\left[F_{\imath \jmath}, B_{\imath}\right], B_{\jmath}\right)\right\} d v o l .
\end{aligned}
$$

Let $D$ be a Riemannian connection of $M$ and let $R$ denote the curvature tensor field of $D ; R\left(e_{\imath}, e_{\jmath}\right) e_{k}=R_{\imath j k l} e_{l}$. The Ricci tensor field Ric of $M$ is defined by $R_{\imath \jmath}=R_{i k k}$. The scalar curvature $R$ of $M$ is defined by $R=R_{i i}$. The Ricci identities are as follows:

$$
\begin{aligned}
& D_{k} D_{\jmath} X^{i}-D_{j} D_{k} X^{i}=R_{k j l \imath} X^{\imath} \quad \text { for } X=X^{i} e_{\imath}, \\
& \nabla_{l} \nabla_{k} F_{\imath j}-\nabla_{k} \nabla_{l} F_{\imath \jmath}=-F_{m j} R_{l k \imath j}-F_{\imath m} R_{l k \jmath}+\left[F_{l k}, F_{\imath \jmath}\right],
\end{aligned}
$$

The curvature form $F^{\nabla}$ always satisfies the Bianchi identity $d^{\nabla} F^{\nabla}=0$, or equivalently

$$
\nabla_{k} F_{\imath j}+\nabla_{\imath} F_{j k}+\nabla_{j} F_{k \imath}=0
$$

The Yang-Mills equation is $\delta^{\nabla} F^{\nabla}=0$, namely

$$
\nabla_{j} F_{\imath \jmath}=0 \text {. }
$$

Let $\nabla \in \mathcal{C}_{E}$. Assume that $\varphi=(1 / 2) \varphi_{i j} \omega^{2} \wedge \omega^{\jmath} \in \Omega^{2}\left(g_{E}\right)$ is harmonic with respect to $\nabla$, that is, $d^{\nabla} \varphi=0$ and $\delta^{\nabla} \varphi=0$. Note that if $\nabla$ is a Yang-Mills connection, we can take $\varphi=F^{\nabla}$. Let $V \in C^{\infty}(T M)$ with $V=V^{i} e_{\imath}$. Set $B=i_{V} \varphi=B_{i} \omega_{i} \in \Omega^{1}\left(g_{E}\right)$. Here $B_{\imath}=V^{\jmath} \varphi_{j i}$. Then by the harmonicity of $\varphi$ and the Bochner-Weitzenböck formula (cf. [B-L]) we compute

$$
\begin{aligned}
\left(\mathcal{S}^{\nabla}(B)\right)(X)= & \varphi\left(D^{*} D V, X\right)-2 \sum_{i=1}^{n}\left(\nabla_{e_{i}} \varphi\right)\left(D_{e_{i}} V, X\right) \\
+ & \varphi(V, \operatorname{Ric}(X))-\{\varphi \circ(\operatorname{Ric} \wedge I-2 \mathscr{R})\}(V, X) \\
& -\sum_{\imath=1}^{n}\left\{\left[F^{\nabla}\left(e_{\imath}, V\right), \varphi\left(e_{\imath}, X\right)\right]+\left[F^{\nabla}\left(e_{\imath}, X\right), \varphi\left(e_{\imath}, V\right)\right]\right\},
\end{aligned}
$$

where $D^{*} D V=-\sum_{\imath=1}^{n} D^{2} V\left(e_{\imath}, e_{\imath}\right)$, and $\mathcal{R}$ denotes the curvature operator of $(M, g)$ acting on $\wedge^{2} T M$. We define a quadratic form $Q_{\varphi}$ on $C^{\infty}(T M)$ as

$$
Q_{\varphi}(V)=\left.\left(d^{2} / d t^{2}\right) q_{\mathcal{M}} \mathscr{M}\left(\nabla^{t}\right)\right|_{t=0}=\int_{M} q_{\varphi}(V) d v o l,
$$

where $\nabla^{t}=\nabla+t\left(i_{V} \varphi\right) \in \mathcal{C}_{E}$. By straightforward computations we have 


$$
\begin{aligned}
q_{\varphi}(V)= & D_{j} D_{i} V^{k} V^{l}\left(\varphi_{k \jmath}, \varphi_{l \imath}\right)-D_{j} D_{j} V^{k} V^{l}\left(\varphi_{k \imath}, \varphi_{l \imath}\right) \\
& +D_{j} V^{k} V^{l}\left(\nabla_{i} \varphi_{k j} \varphi_{l \imath}\right)-2 D_{j} V^{k} V^{l}\left(\nabla_{j} \varphi_{k \imath}, \varphi_{l \imath}\right) \\
& +V^{k} V^{l}\left(\left[F_{j k}^{\nabla}, \varphi_{i \jmath}\right]+\left[F_{j i}^{\nabla}, \varphi_{k \jmath}\right], \varphi_{l \imath}\right) \\
& +V^{k} V^{l}\left\{R_{i_{k} j}\left(\varphi_{m \jmath}, \varphi_{l \imath}\right)-R_{j i k m}\left(\varphi_{m \jmath}, \varphi_{l \imath}\right)+R_{k m}\left(\varphi_{i m}, \varphi_{l \imath}\right)\right\} .
\end{aligned}
$$

\section{The constructionn of Ruh for a $\delta$-pinched manifold.}

We recall the idea and construction of Ruh ([Ru], [G-K-R1], [G-K-R2]). Let $(M, g)$ be an $n$-dimensional simply connected compact Riemannian manifold with $\delta$-pinched sectional curvature, namely $\delta<K \leqq 1$. We fix a normalized Riemannian metric $g_{0}=\{(1+\delta) / 2\} g$ on $M$. Then we have $2 \delta /(1+\delta)<K_{g_{0}} \leqq 2 /(1+\delta)$. Consider a vector bundle $\Xi=T M \oplus \varepsilon(M)$ with a fibre metric $\langle$, $\rangle$ over $M$. Here $\varepsilon(M)$ is a trivial line bundle with a fiber metric and it is orthogonal to $T M$. Let $e$ denote a smooth section of lengh 1 in $\varepsilon(M)$. Now we define a metric connection $D^{\prime \prime}$ in $\boldsymbol{\Xi}$ as follows;

$$
\begin{aligned}
& D_{X}^{\prime \prime} Y=D_{X} Y-g_{0}(X, Y) e, \\
& D_{X}^{\prime \prime} e=X
\end{aligned}
$$

for $X, Y \in C^{\infty}(T M)$. It was proved that if $\delta$ is sufficiently close to 1 , there exists a flat connection $D^{\prime}$ in $\Xi$ close to $D^{\prime \prime}$ ([G-K-R1]). Define

$$
\left\|D^{\prime}-D^{\prime \prime}\right\|:=\underset{x \in M}{\operatorname{Ma}} x\left\{\left\|D_{X}^{\prime} Y-D_{X}^{\prime \prime} Y\right\| ; X \in T_{x} M, g_{0}(X, X)=1, Y \in \Xi_{x},\|Y\|=1\right\} .
$$

Note that it is a half of that one in [G-K-R2]. Set

$$
\begin{aligned}
& k_{1}(\delta)=(4 / 3)(1-\delta) \delta^{-1}\left\{1+\left(\delta^{1 / 2} \sin (1 / 2) \pi \delta^{-1 / 2}\right)^{-1}\right\}, \\
& k_{2}(\delta)=\{(1+\delta) / 2\}^{-1} k_{1}(\delta), \\
& k_{3}(\delta)=k_{2}(\delta)\left[1+\left\{1-(1 / 24) \pi^{2} k_{2}(\delta)^{2}\right\}^{-2}\right]^{1 / 2} .
\end{aligned}
$$

[G-K-M2] proved that $\left\|D^{\prime}-D^{\prime \prime}\right\| \leqq k_{3}(\delta) / 2$. The curvature form $R^{\prime \prime}$ of the connection $D^{\prime \prime}$ is

$$
\begin{aligned}
& R^{\prime \prime}(X, Y) Z=R(X, Y) Z-\langle Y, Z\rangle X+\langle X, Z\rangle Y, \\
& R^{\prime \prime}(X, Y) e=0
\end{aligned}
$$

for $X, Y, Z \in T_{x} M$. 


\section{Trace formula for second variations of Yang-Mills fields over a $\delta$-pinched manifold.}

Assume that $M$ is a simply connected compact Riemannian manifold with $\delta$-pinched sectional curvatures. Let $P=\left\{v \in C^{\infty}(\boldsymbol{\Xi}) ; D^{\prime} v=0\right\}$, which is linerly isometric to $\boldsymbol{R}^{n+1}$. For each $v \subseteq P$, we denote by $V=v^{T}$ the $T M$-component of $v$ in $\Xi$. Set $\mathscr{V}=\left\{V \in C^{\infty}(T M) ; V=v^{T}\right.$ for some $\left.v \in P\right\}$, which has a natural inner product so that it is linearly isometric to $P$. Choose an orthonormal basis $\left\{V_{\alpha}\right\}_{\alpha=0, \ldots, n}$ of $\mathcal{C}$. Set $V_{\alpha}=\left(v_{\alpha}\right)^{T}$. Then $\sum_{\alpha=0}^{n} V_{\alpha}^{k} V_{\alpha}^{\iota}=\delta^{k l}$. In this section we compute the trace $\operatorname{Tr}_{\alpha} Q_{\varphi}=\sum_{\alpha=0}^{n} Q_{\varphi}\left(V_{\alpha}\right)$ of $Q_{\varphi}$ on $\mathcal{V}$ relative to the inner product.

A straightforward computation shows

LEMMA 3.1 .

$$
\begin{aligned}
& D_{j} V^{k}=\left\langle D_{e_{j}}^{\prime \prime} v, e_{k}\right\rangle-\langle v, e\rangle \delta_{j k} . \\
& D_{j} D_{i} V^{k}=\left\langle\left(D^{\prime \prime 2} v\right)\left(e_{\imath}, e_{j}\right), e_{k}\right\rangle-\delta_{j k}\left\langle D_{e_{i}}^{\prime \prime} v, e\right\rangle-\delta_{i k}\left\langle D_{e_{j}}^{\prime \prime} v, e\right\rangle-\delta_{i k}\left\langle v, e_{\jmath}\right\rangle .
\end{aligned}
$$

LEMMA 3.2.

$$
\begin{aligned}
& \int_{M}\left\{D_{j} D_{i} V^{k} V^{l}\left(\varphi_{k \jmath}, \varphi_{\imath \imath}\right)+D_{j} V^{k} V^{l}\left(\nabla_{i} \varphi_{k \jmath}, \varphi_{l \imath}\right)\right\} d v o l \\
= & \int_{M}\left\{R_{j i m k} V^{m} V_{\imath}\left(\varphi_{k \jmath}, \varphi_{l \imath}\right)-D_{j} V^{k} D_{\imath} V^{l}\left(\left(\varphi_{k \jmath}, \varphi_{l \imath}\right)\right\} d v o l .\right. \\
& \int_{M}-2 D_{j} V_{\alpha}^{k} V_{\alpha}^{\iota}\left(\nabla_{j} \varphi_{k \imath}, \varphi_{l \imath}\right) d v o l \\
= & \int_{M}\left\{-2 D_{k} D_{j} V_{\alpha}^{k} V_{\alpha}^{\iota}\left(\varphi_{i \jmath}, \varphi_{l \imath}\right)-2 D_{j} V_{\alpha}^{k} D_{k} V_{\alpha}^{\iota}\left(\varphi_{i \jmath}, \varphi_{l \imath}\right)\right. \\
& -D_{i} D_{j} V_{\alpha}^{k} V_{\alpha}^{\iota}\left(\varphi_{i \jmath}, \varphi_{k \imath}\right)-D_{\jmath} V_{\alpha}^{k} D_{\imath} V_{\alpha}^{l}\left(\varphi_{i \jmath}, \varphi_{k \imath}\right) \\
& \left.-2 D_{\imath} D_{j} V_{\alpha}^{k} V_{\alpha}^{\iota}\left(\varphi_{j k}, \varphi_{l \imath}\right)-2 D_{j} V_{\alpha}^{k} D_{\imath} V_{\alpha}^{l}\left(\varphi_{j k}, \varphi_{l \imath}\right)\right\} d v o l .
\end{aligned}
$$

Proof. (3.3) is due to the Ricci identity and the divergence theorem. We show (3.4). By $d^{\nabla} \varphi=0$, we have

$$
\begin{aligned}
& -2 D_{j} V_{\alpha}^{k} V_{\alpha}^{l}\left(\nabla_{j} \varphi_{k \imath}, \varphi_{l \imath}\right) \\
= & 2 D_{j} V_{\alpha}^{k} V_{\alpha}^{l}\left(\nabla_{k} \varphi_{i \jmath}, \varphi_{\imath \imath}\right)+2 D_{j} V_{\alpha}^{k} V_{\alpha}^{l}\left(\nabla_{i} \varphi_{j k}, \varphi_{\imath \imath}\right),
\end{aligned}
$$

By using the divergence theorem, we get

$$
\int_{M} 2 D_{j} V_{\alpha}^{k} V_{\alpha}^{l}\left(\nabla_{i} \varphi_{j k}, \varphi_{l \imath}\right) d v o l
$$




$$
=\int_{M}\left\{-2 D_{i} D_{j} V_{\alpha}^{k} V_{\alpha}^{l}\left(\varphi_{j k}, \varphi_{l \imath}\right)-2 D_{j} V_{\alpha}^{k} D_{i} V_{\alpha}^{\iota}\left(\varphi_{j k}, \varphi_{l \imath}\right)\right\} d v o l .
$$

We compute

Since

$$
\begin{aligned}
& 2 D_{j} V_{\alpha}^{k} V_{\alpha}^{l}\left(\nabla_{k} \varphi_{i \jmath}, \varphi_{\imath \imath}\right) \\
= & 2 D_{k}\left\{D_{j} V_{\alpha}^{k} V_{\alpha}^{l}\left(\varphi_{i \jmath}, \varphi_{\imath \imath}\right)\right\}-2 D_{k} D_{j} V_{\alpha}^{k} V_{\alpha}^{l}\left(\varphi_{i \jmath}, \varphi_{l \imath}\right) \\
& -2 D_{j} V_{\alpha}^{k} D_{k} V_{\alpha}^{l}\left(\varphi_{i \jmath}, \varphi_{l \imath}\right)-2 D_{j} V_{\alpha}^{k} V_{\alpha}^{l}\left(\varphi_{i \jmath}, \nabla_{k} \varphi_{l \imath}\right) .
\end{aligned}
$$

$$
D_{j} V_{\alpha}^{k} V_{\alpha}^{l}=-V_{\alpha}^{k} D_{j} V_{\alpha}^{l}
$$

we have

$$
D_{\jmath} V_{\alpha}^{k} V_{\alpha}^{l}\left(\varphi_{i \jmath}, \nabla_{k} \varphi_{l \imath}\right)=D_{j} V_{\alpha}^{k} V_{\alpha}^{l}\left(\varphi_{i \jmath}, \nabla_{l} \varphi_{i k}\right) .
$$

Hence by Bianchi identity we get

$$
-2 D_{j} V_{\alpha}^{k} V_{\alpha}^{k}\left(\varphi_{i j}, \nabla_{k} \varphi_{\imath \imath}\right)=D_{j} V_{\alpha}^{k} V_{\alpha}^{\iota}\left(\varphi_{i j}, \nabla_{i} \varphi_{k l}\right) .
$$

Thus by using the divergence theorem we obtain

$$
\begin{aligned}
& \int_{M} 2 D_{j} V_{\alpha}^{k} V_{\alpha}^{l}\left(\nabla_{k} \varphi_{i \jmath}, \varphi_{l \imath}\right) d v o l \\
= & \int_{M}\left\{-2 D_{k} D_{j} V_{\alpha}^{k} V_{\alpha}^{\iota}\left(\varphi_{i \jmath}, \varphi_{l \imath}\right)-2 D_{j} V_{\alpha}^{k} D_{k} V_{\alpha}^{l}\left(\varphi_{i \jmath}, \varphi_{l \imath}\right)\right. \\
& \left.-D_{\imath} D_{j} V_{\alpha}^{k} V_{\alpha}^{l}\left(\varphi_{i \jmath}, \varphi_{k l}\right)-D_{j} V_{\alpha}^{k} D_{i} V_{\alpha}^{\iota}\left(\varphi_{i \jmath}, \varphi_{k l}\right)\right\} d v o l .
\end{aligned}
$$

By (1.5), (3.3) and (3.4), we get

$$
\begin{aligned}
\operatorname{Tr}_{\iota} Q_{\varphi}= & \int_{M}\left\{-D_{j} V_{\alpha}^{k} D_{\imath} V_{\alpha}^{\imath}\left(\varphi_{k \jmath}, \varphi_{\imath \imath}\right)-D_{j} D_{j} V_{\alpha}^{k} V_{\alpha}^{\iota}\left(\varphi_{k \imath}, \varphi_{\imath \imath}\right)\right. \\
& -2 D_{k} D_{j} V_{\alpha}^{k} V_{\alpha}^{\iota}\left(\varphi_{i \jmath}, \varphi_{\imath \imath}\right)-2 D_{j} V_{\alpha}^{k} D_{k} V_{\alpha}^{\imath}\left(\varphi_{i \jmath}, \varphi_{\imath \imath}\right) \\
& -D_{\imath} D_{j} V_{\alpha}^{k} V_{\alpha}^{\iota}\left(\varphi_{i \jmath}, \varphi_{k \imath}\right)-D_{j} V_{\alpha}^{k} D_{i} V_{\alpha}^{\iota}\left(\varphi_{i \jmath}, \varphi_{k \imath}\right) \\
& -2 D_{\imath} D_{\jmath} V_{\alpha}^{k} V_{\alpha}^{\iota}\left(\varphi_{j k}, \varphi_{l \imath}\right)-2 D_{j} V_{\alpha}^{k} D_{\imath} V_{\alpha}^{\iota}\left(\varphi_{j k}, \varphi_{\imath \imath}\right) \\
& +R_{j i l k}\left(\varphi_{k \jmath}, \varphi_{\imath \imath}\right)+R_{i k m j}\left(\varphi_{m \jmath}, \varphi_{k \imath}\right) \\
& \left.-R_{j i k m}\left(\varphi_{m \jmath}, \varphi_{k \imath}\right)+R_{k m}\left(\varphi_{i m}, \varphi_{k \imath}\right)\right\} d v o l .
\end{aligned}
$$

LEMMA 3.3.

$$
\begin{aligned}
& -2 D_{i} D_{j} V_{\alpha}^{k} V_{\alpha}^{l}\left(\varphi_{j k}, \varphi_{l \imath}\right) \\
= & D_{j} V_{\alpha}^{k} D_{i} V_{\alpha}^{l}\left(\varphi_{j k}, \varphi_{l \imath}\right)+D_{i} V_{\alpha}^{k} D_{j} V_{\alpha}^{l}\left(\varphi_{j k}, \varphi_{l \imath}\right) \\
& +R_{j i m k} V_{\alpha}^{m} V_{\alpha}^{l}\left(\varphi_{j k}, \varphi_{l \imath}\right),
\end{aligned}
$$




$$
-D_{i} D_{j} V_{\alpha}^{k} V_{\alpha}^{\iota}\left(\varphi_{i \jmath}, \varphi_{k l}\right)=-(1 / 2) R_{\imath \jmath m k} V_{\alpha}^{m} V_{\alpha}^{\iota}\left(\varphi_{i \jmath}, \varphi_{k l}\right) \text {. }
$$

Proof. (3.9) is due to the Ricci identity. We show (3.8). Differentiating covariantly (3.6), we have

$$
D_{i} D_{j} V_{\alpha}^{k} V_{\alpha}^{\iota}+V_{\alpha}^{k} D_{i} D_{j} V_{\alpha}^{\iota}+D_{j} V_{\alpha}^{k} D_{\imath} V_{\alpha}^{\iota}+D_{\imath} V_{\alpha}^{k} D_{j} V_{\alpha}^{l}=0 .
$$

(3.8) follows from (3.10) and the Ricci identity.

q.e.d.

LEMMA 3.4.

$$
\begin{aligned}
-D_{j} D_{j} V_{\alpha}^{k} V_{\alpha}^{\iota}\left(\varphi_{k \imath}, \varphi_{\imath \imath}\right)= & \left\langle D_{e_{j}}^{\prime \prime} v_{\alpha}, D_{e_{i}}^{\prime \prime} v_{\beta}\right\rangle V_{\beta}^{k} V_{\alpha}^{\iota}\left(\varphi_{k \imath}, \varphi_{l \imath}\right) \\
& +\left\{2\left\langle D_{e_{k}}^{\prime \prime} v_{\alpha}, e\right\rangle+\left\langle v_{\alpha}, e_{k}\right\rangle\right\} V_{\alpha}^{\iota}\left(\varphi_{k \imath}, \varphi_{l \imath}\right)
\end{aligned}
$$

Proof. From $\left\langle v_{\alpha}, v_{\beta}\right\rangle=\delta_{\alpha \beta}$, we have

$$
\begin{aligned}
& \left\langle\left(D^{\prime \prime 2} v_{\alpha}\right)\left(e_{\imath}, e_{j}\right), v_{\beta}\right\rangle+\left\langle\left(D^{\prime \prime 2} v_{\beta}\right)\left(e_{\imath}, e_{j}\right), v_{\alpha}\right\rangle \\
= & -\left\langle D_{e_{i}}^{\prime \prime} v_{\alpha}, D_{e_{j}}^{\prime \prime} v_{\beta}\right\rangle-\left\langle D_{e_{j}}^{\prime \prime} v_{\alpha}, D_{e_{i}}^{\prime \prime} v_{\beta}\right\rangle .
\end{aligned}
$$

Using (3.2) and (3.12), we obtain (3.11).

q.e.d.

\section{LEMMA 3.5.}

$$
\begin{aligned}
\int_{M} & -2 D_{k} D_{j} V_{\alpha}^{k} V_{\alpha}^{\iota}\left(\varphi_{i \jmath}, \varphi_{l \imath}\right) d v o l \\
=\int_{M} & {\left[2\left\langle D_{e_{j}}^{\prime \prime} v_{\alpha}, e\right\rangle V_{\alpha}^{l}\left(\varphi_{i_{\jmath}}, \varphi_{l \imath}\right)\right.} \\
+ & 2\left\langle D_{e_{k}}^{\prime \prime} v_{\alpha}, e_{k}\right\rangle\left\langle D_{e_{j}}^{\prime \prime} v_{\alpha}, e_{l}\right\rangle\left(\varphi_{i \jmath}, \varphi_{l \imath}\right) \\
+ & 2\left\{(2-(n / 2))\left\langle D_{e_{k}}^{\prime \prime} v_{\alpha}, e_{k}\right\rangle\left\langle v_{\alpha}, e\right\rangle-(1 / 4)\left\langle R^{\prime \prime}\left(e_{l}, e_{k}\right) e_{k}, e_{l}\right\rangle\right. \\
& -(1 / 4)\left\langle D_{e_{k}}^{\prime \prime} v_{\alpha}, D_{e_{l}}^{\prime \prime} v_{\beta}\right\rangle\left\langle v_{\beta}, e_{k}\right\rangle\left\langle v_{\alpha}, e_{l}\right\rangle \\
& -(1 / 4)\left\langle D_{e_{k}}^{\prime \prime} v_{\alpha}, D_{e_{l}}^{\prime \prime} v_{\beta}\right\rangle\left\langle v_{\beta}, e_{l}\right\rangle\left\langle v_{\alpha}, e_{k}\right\rangle \\
& \left.-(1 / 2)\left\langle D_{e_{k}}^{\prime \prime} v_{\alpha}, e\right\rangle V_{\alpha}^{k}+(1 / 2)\left\langle D_{e_{k}}^{\prime \prime} v_{\alpha}, e_{k}\right\rangle\left\langle D_{e_{l}}^{\prime \prime} v_{\alpha}, e_{l}\right\rangle\right\}\|\varphi\|^{2} \\
- & 2\left\langle R^{\prime \prime}\left(e_{k}, e_{j}\right) e_{l}, e_{k}\right\rangle\left(\varphi_{i \jmath}, \varphi_{l \imath}\right)+2(n+1)\left\langle D_{e_{j}}^{\prime \prime} v_{\alpha}, e\right\rangle V_{\alpha}^{\iota}\left(\varphi_{i \jmath}, \varphi_{l \imath}\right) \\
+ & \left.2\left\langle v_{\alpha}, e_{\jmath}\right\rangle V_{\alpha}^{l}\left(\varphi_{i \jmath}, \varphi_{l \imath}\right)\right] d v o l .
\end{aligned}
$$

Proof. By (3.2), we have

$$
\begin{aligned}
& -2 D_{k} D_{j} V_{\alpha}^{k} V_{\alpha}^{\iota}\left(\varphi_{i \jmath}, \varphi_{\imath \imath}\right) \\
= & -2\left\{\left\langle\left(D^{\prime \prime 2} v_{\alpha}\right)\left(e_{\jmath}, e_{k}\right), e_{k}\right\rangle-(n+1)\left(D_{e_{j}}^{\prime \prime} v_{\alpha}, e\right\rangle\right. \\
& \left.-\left\langle v_{\alpha}, e_{\jmath}\right\rangle\right\} V_{\alpha}^{l}\left(\varphi_{i \jmath}, \varphi_{l \imath}\right) .
\end{aligned}
$$


By using the Ricci identity we get

$$
\begin{aligned}
& \left\langle\left(D^{\prime \prime 2} v_{\alpha}\right)\left(e_{\jmath}, e_{k}\right), e_{k}\right\rangle V_{\alpha}^{\iota}\left(\varphi_{i \jmath}, \varphi_{l \imath}\right) \\
= & \left\{\left\langle\left(D^{\prime \prime 2} v_{\alpha}\right)\left(e_{k}, e_{j}\right), e_{k}\right\rangle+\left\langle R^{\prime \prime}\left(e_{k}, e_{j}\right) v_{p}, e_{k}\right\rangle\right\} V_{\alpha}^{l}\left(\varphi_{i \jmath}, \varphi_{l \imath}\right) .
\end{aligned}
$$

We compute

$$
\begin{aligned}
& \left\langle\left(D^{\prime \prime 2} v_{\alpha}\right)\left(e_{k}, e_{j}\right), e_{k}\right\rangle V_{\alpha}^{l}\left(\varphi_{i \jmath}, \varphi_{l \imath}\right) \\
= & D_{j}\left\{\left\langle D_{e_{k}}^{\prime \prime} v_{\alpha}, e_{k}\right\rangle V_{\alpha}^{l}\left(\varphi_{i \jmath}, \varphi_{l \imath}\right)\right\}-\left\langle D_{e_{j}}^{\prime \prime} v_{\alpha}, e\right\rangle V_{\alpha}^{l}\left(\varphi_{i \jmath}, \varphi_{l \imath}\right) \\
& -\left\langle D_{e_{k}}^{\prime \prime} v^{o}, e_{k}\right\rangle\left\langle D_{e_{i}}^{\prime \prime} v_{\alpha}, e_{l}\right\rangle\left(\varphi_{i \jmath}, \varphi_{l \imath}\right)-\left\langle D_{e_{k}}^{\prime \prime} v_{\alpha}, e_{k}\right\rangle\left\langle v_{\alpha}, e\right\rangle\left(\varphi_{i \jmath}, \varphi_{i j}\right) \\
& -\left\langle D_{e_{k}}^{\prime \prime} v_{\alpha}, e_{k}\right\rangle V_{\alpha}^{l}\left(\varphi_{i \jmath}, \nabla_{j} \varphi_{\imath \imath}\right) .
\end{aligned}
$$

By the Bianchi identity we get

$$
-\left\langle D_{e_{k}}^{\prime \prime} v_{\alpha}, e_{k}\right\rangle V_{\alpha}^{\iota}\left(\varphi_{i \jmath}, \nabla_{j} \varphi_{l \imath}\right)=(1 / 4)\left\langle D_{e_{k}}^{\prime \prime} v_{\alpha}, e_{k}\right\rangle V_{\alpha}^{\iota} D_{l}\|\varphi\|^{2} .
$$

We compute

$$
\begin{aligned}
& \left\langle D_{e_{k}}^{\prime \prime} v_{\alpha}, e_{k}\right\rangle V_{\alpha}^{\iota} D_{l}\|\varphi\|^{2} \\
= & D_{l}\left\{\left\langle D_{e_{k}}^{\prime \prime} v_{\alpha}, e_{k}\right\rangle V_{\alpha}^{\iota}\|\varphi\|^{2}\right\}-\left\langle\left(D^{\prime \prime 2} v_{\alpha}\right)\left(e_{k}, e_{l}\right), e_{k}\right\rangle V_{\alpha}^{\iota}\|\varphi\|^{2} \\
& +\left\langle D_{e_{k}}^{\prime \prime} v_{\alpha}, e\right\rangle V_{\alpha}^{k}\|\varphi\|^{2}-\left\langle D_{e_{k}}^{\prime \prime} v_{\alpha}, e_{k}\right\rangle\left\langle D_{e_{l}}^{\prime \prime} v_{\alpha}, e_{l}\right\rangle\|\varphi\|^{2} \\
& +n\left\langle D_{e_{k}}^{\prime \prime} v_{\alpha}, e_{k}\right\rangle\left\langle v_{\alpha}, e\right\rangle\|\varphi\|^{2} .
\end{aligned}
$$

By using (3.12) and the Ricci identity we get

$$
\begin{aligned}
& \left\langle\left(D^{\prime \prime 2} v_{\alpha}\right)\left(e_{k}, e_{l}\right), e_{k}\right\rangle V_{\alpha}^{\iota} \\
= & -(1 / 2)\left\{\left\langle R^{\prime \prime}\left(e_{l}, e_{k}\right) e_{k}, e_{l}\right\rangle+\left\langle D_{e_{k}}^{\prime \prime} v_{\alpha}, D_{e_{l}}^{\prime \prime} v_{\beta}\right\rangle V_{\alpha}^{k} V_{\alpha}^{\iota}\right. \\
& \left.+\left\langle D_{e_{k}}^{\prime \prime} v_{\alpha}, D_{e_{l}}^{\prime \prime} v_{\beta}\right\rangle V_{\beta}^{\iota} V_{\alpha}^{k}\right\} .
\end{aligned}
$$

Hence, by the divergence theorem, (3.13) follows from (3.14), (3.15), (3.16), (3.17), (3.16) and (3.19).

q.e.d.

Therefore, by (2.1), (3.8), (3.11) and (3.13), (3.7) reduces to the following trace formula.

$$
\begin{aligned}
& \operatorname{Tr}_{\mathcal{V}} Q_{\varphi} \\
= & \int_{M}\left[2\{5-2 n+(n(n-1)-R) / 4\}\|\varphi\|^{2}+R_{j l}\left(\varphi_{i \jmath}, \varphi_{i l}\right)\right. \\
& +\left\langle D_{e_{i}}^{\prime \prime} v_{\alpha}, D_{e_{i}}^{\prime \prime} v_{\beta}\right\rangle V_{\beta}^{k} V_{\alpha}^{l}\left(\varphi_{k \imath}, \varphi_{l \imath}\right)-2\left\langle D_{e_{k}}^{\prime \prime} v_{\alpha}, e_{k}\right\rangle\left\langle D_{e_{j}}^{\prime \prime} v_{\alpha}, e_{l}\right\rangle\left(\varphi_{i \jmath}, \varphi_{i l}\right)
\end{aligned}
$$




$$
\begin{aligned}
& +2\left\{(2-\langle n / 2))\left\langle D_{e_{k}}^{\prime \prime} v_{\alpha}, e_{k}\right\rangle\left\langle v_{\alpha}, e\right\rangle\right. \\
& \quad-(1 / 4)\left\langle D_{e_{k}}^{\prime \prime} v_{\alpha}, D_{e_{l}}^{\prime \prime} v_{\beta}\right\rangle\left\langle v_{\beta}, e_{k}\right\rangle\left\langle v_{\alpha}, e_{l}\right\rangle \\
& \quad-(1 / 4)\left\langle D_{e_{k}}^{\prime \prime} v_{\alpha}, D_{e_{l}}^{\prime \prime} v_{\beta}\right\rangle\left\langle v_{\beta}, e_{l}\right\rangle\left\langle v_{\alpha}, e_{k}\right\rangle \\
& \left.\quad-(1 / 2)\left\langle D_{e_{k}}^{\prime \prime} v_{\alpha}, e\right\rangle V_{\alpha}^{k}+(1 / 2)\left\langle D_{e_{k}}^{\prime \prime} v_{\alpha}, e_{k}\right\rangle\left\langle D_{e_{l}}^{\prime \prime} v_{\alpha}, e_{l}\right\rangle\right\}\|\varphi\|^{2} \\
& -2(n+1)\left\langle D_{e_{j}}^{\prime \prime} v_{\alpha}, e\right\rangle V_{\alpha}^{\iota}\left(\varphi_{i \jmath}, \varphi_{i l}\right)-8\left\langle D_{e_{j}}^{\prime \prime} v_{\alpha}, e_{k}\right\rangle\left\langle v_{\alpha}, e\right\rangle\left(\varphi_{i \jmath}, \varphi_{i_{k}}\right) \\
& +2\left\langle D_{e_{j}}^{\prime \prime} v_{\alpha}, e_{k}\right\rangle\left\langle D_{e_{k}}^{\prime \prime} v_{\alpha}, e_{l}\right\rangle\left(\varphi_{i \jmath}, \varphi_{i l}\right)-\left\langle D_{e_{j}}^{\prime \prime} v_{\alpha}, e_{k}\right\rangle\left\langle D_{e_{i}}^{\prime \prime} v_{\alpha}, e_{l}\right\rangle\left(\varphi_{i \jmath}, \varphi_{k l}\right) \\
& \left.+\left\langle D_{e_{l}}^{\prime \prime} v_{\alpha}, e_{\jmath}\right\rangle\left\langle D_{e_{i}}^{\prime \prime} v_{\alpha}, e_{k}\right\rangle\left(\varphi_{i_{\jmath}}, \varphi_{k l}\right)\right] d v o l .
\end{aligned}
$$

\section{Instability theorem for Yang-Millf fields} over a $\delta$-pinched Riemannian manifold.

Note that if $\delta=1$, then $D^{\prime}=D^{\prime \prime}$, hence (3.20) becomes

$$
\operatorname{Tr}_{C \nu} Q_{\varphi}=2(4-n) \int_{M}\|\varphi\|^{2}
$$

Since the sectional curvatures of $M$ are $\delta$-pinched, we have

$$
\begin{aligned}
& 2\{5-2 n+(1 / 4)(n(n-1)-R)\}\|\varphi\|^{2}+R_{j l}\left(\varphi_{i \jmath}, \varphi_{i l}\right) \\
\leqq & 2[5-2 n+(1 / 4) n(n-1)\{1-2 \delta /(1+\delta)\}+2(n-1) /(1+\delta)]\|\varphi\|^{2},
\end{aligned}
$$

We can make estimates for each other term of (3.20) as follows:

$$
\begin{aligned}
& \left\langle D_{e_{i}}^{\prime \prime} v_{\alpha}, D_{e_{i}}^{\prime \prime} v_{\beta}\right\rangle V_{\beta}^{l} V_{\alpha}^{\iota}\left(\varphi_{k \imath}, \varphi_{l \imath}\right) \leqq(n / 2) k_{3}(\delta)^{2}\|\varphi\|^{2}, \\
& -2\left\langle D_{e_{l}}^{\prime \prime} v_{\alpha}, e_{k}\right\rangle\left\langle D_{e_{j}}^{\prime \prime} v_{\alpha}, e_{l}\right\rangle\left(\varphi_{i j}, \varphi_{i l}\right) \leqq n(n+1) k_{3}(\delta)^{2}\|\varphi\|^{2}, \\
& (2-(n / 2))\left\langle D_{e_{k}}^{\prime \prime} v_{\alpha}, e_{k}\right\rangle\left\langle v_{\alpha}, e\right\rangle \leqq n(n / 4-1) k_{3}(\delta), \\
& -(1 / 4)\left\langle D_{e_{k}}^{\prime \prime} v_{\alpha}, D_{e_{l}}^{\prime \prime} v_{\beta}\right\rangle\left\langle v_{\beta}, e_{k}\right\rangle\left\langle v_{\alpha}, e_{l}\right\rangle \leqq\left(n^{2} / 16\right) k_{3}(\delta)^{2}, \\
& -(1 / 4)\left\langle D_{e_{k}}^{\prime \prime} v_{\alpha}, D_{e_{l}}^{\prime \prime} v_{\beta}\right\rangle\left\langle v_{\beta}, e_{l}\right\rangle\left\langle v_{\alpha}, e_{k}\right\rangle \leqq\left(n^{2} / 16\right) k_{3}(\delta)^{2}, \\
& -(1 / 2)\left\langle D_{e_{k}}^{\prime \prime} v_{\alpha}, e\right\rangle V_{\alpha}^{k} \leqq(n / 4) k_{3}(\delta), \\
& -2(n+1)\left\langle D_{e_{k}}^{\prime \prime} v_{\alpha}, e_{k}\right\rangle\left\langle D_{e_{l}}^{\prime \prime} v_{\alpha}, e_{l}\right\rangle \leqq\left(n^{2} / 8\right) k_{3}(\delta)^{2}, \\
& -8\left\langle D_{e_{j}}^{\prime \prime} v_{\alpha}, e_{k}\right\rangle\left\langle\varphi_{i j}, \varphi_{i l}\right) \leqq 2(n+1) k_{3}(\delta)\|\varphi\|^{2}, \\
& 2\left\langle D_{e_{j}}^{\prime \prime} v_{\alpha}, e_{k}\right\rangle\left\langle D_{e_{k}}^{\prime \prime} v_{\alpha}, e_{l}\right\rangle\left(\varphi_{i j}, \varphi_{i l}\right) \leqq n k_{3}(\delta)\|\varphi\|^{2}, \\
& \left\langle D_{e_{l}}^{\prime \prime} v_{\alpha}, e_{j}\right\rangle\left\langle D_{e_{i}}^{\prime \prime} v_{\alpha}, e_{k}\right\rangle\left(\varphi_{i j}, \varphi_{k l}\right) \\
& \quad-\left\langle D_{e_{j}}^{\prime \prime} v_{\alpha}, e_{k}\right\rangle\left\langle D_{e_{i}}^{\prime \prime} v_{\alpha}, e_{l}\right\rangle\left(\varphi_{i \jmath}, \varphi_{k l}\right) \leqq k_{3}(\delta)\|\varphi\|^{2} .
\end{aligned}
$$


Hence we get

$$
\begin{aligned}
\operatorname{Tr}_{\mathcal{V}} Q_{\varphi} \leqq & 2[5-2 n+(1 / 4) n(n-1)\{1-2 \delta /(1+\delta)\}+2(n-1) /(1+\delta) \\
& \left.+(1 / 4)\left(n^{2}+n+20\right) k_{3}(\delta)+(1 / 4)\left(3 n^{2}+5 n+2\right) k_{3}(\delta)^{2}\right] \int_{M}\|\varphi\|^{2}
\end{aligned}
$$

Therefore we obtain

THEOREM 4.1. If $n \geqq 5$ and

$$
\begin{aligned}
& 5-2 n+(1 / 4) n(n-1)\{1-2 \delta /(1+\delta)\}+2(n-1) /(1+\delta) \\
& \quad+(1 / 4)\left(n^{2}+n+20\right) k_{3}(\delta)+(1 / 4)\left(3 n^{2}+5 n+2\right) k_{3}(\delta)^{2}<0,
\end{aligned}
$$

then $M$ is Yang-Mills unstable.

COROLLARY 4.2. For $n \geqq 5$, there exists a constant $\delta(n)$, which depends only on $n$, with $1 / 4<\delta(n)<1$ such that any $n$-dimensional simply connected compact Riemannian manifold $M$ with $\delta(n)$-pinched sectional curvatures is Yang-Mills unstable.

Remark. As $n$ tends to the infinity, the right hand side of (4.2) divided by $(1 / 4)\left(3 n^{2}+5 n+2\right)$ tends to $(1 / 3)\{1-2 \delta /(1+\delta)\}+(1 / 3) k_{3}(\delta)+k_{3}(\delta)^{2}>0$. In our argument it is not possible to find a pinching constant $\delta$ independent of the dimension of the base manifold $M$ such that $M$ is Yang-Mills unstable.

\section{Trace formula for second variations of Yang-Mills fields over submanifolds in Euclidean space.}

Assume that $M$ is isometrically immersed in a Euclidean space $\boldsymbol{R}^{N}$. Let $\Phi$ denote the immersion. We may assume that $\Phi(M)$ is not contained in any hyperplane of $\boldsymbol{R}^{N}$. Set $U=\left\{U \in C^{\infty}(T M) ; U=\operatorname{grad} f_{u}\right.$ for some $\left.u \in \boldsymbol{R}^{N}\right\}$. Here $f_{u}$ denotes the hight function on $M$ defined by $f_{u}(u)=\langle\Phi(x), u\rangle$. Suppose that $\nabla$ is a connection on a Riemannian vector bundle $(E, G)$ over $M$ and $\varphi \in \Omega^{2}\left(g_{E}\right)$ is harmonic with respect to $\nabla$. Then we recall

Proposition 5.1 ([K-O-T]). For $U=\operatorname{grad} f_{u} \in q$,

$$
\begin{aligned}
\mathcal{S}^{\nabla}\left(i_{U} \varphi\right)(X)= & -\{\varphi \circ(\operatorname{Ric} \wedge I-2 R)\}(U, X) \\
& +n \varphi\left(A_{\eta}(U), X\right)+\varphi(U, \operatorname{Ric}(X))-\varphi(\operatorname{Ric}(U), X) \\
& -\sum_{i=1}^{n}\left\{\left[F^{\nabla}\left(e_{\imath}, U\right), \varphi\left(e_{\imath}, X\right)\right]+\left[F^{\nabla}\left(e_{\imath}, X\right), \varphi\left(e_{\imath}, U\right)\right]\right\} \\
& -2 \sum_{\imath, j=1}^{n}\left\langle B\left(e_{\imath}, e_{j}\right), u\right\rangle\left(\nabla_{e_{j}} \varphi\right)\left(e_{\imath}, X\right)-n \sum_{i=1}^{n}\left\langle D^{\perp}{ }_{e_{i}} \eta, \mu\right\rangle \varphi\left(e_{\imath}, X\right) .
\end{aligned}
$$




$$
\operatorname{tr}_{\vartheta} Q_{\varphi}=2 \int_{M}\left(\varphi \cdot\left\{(n / 2)\left(A_{\eta} \wedge I\right)-\operatorname{Ric} \wedge I+2 \mathscr{R}\right\}, \varphi\right) d v o l,
$$

where $\mathcal{R}, B, A, \eta$ and $D^{\perp}$ denote the curvature operator of $M$ acting on $\wedge^{2} T M$, the second fundamental form, the shape operator, the mean curvature and the normal connection of $\Phi$, respectively.

Consider a compact Riemannian homogeneous space with irreducible isotropy representation $M$.

LEMMA 5.2. If $\nabla$ is a weakly stable Yang-Mills connection, then we have

$$
\sum_{\imath=1}^{n}\left\{\left[F^{\nabla}\left(e_{\imath}, Y\right), \varphi\left(e_{\imath}, X\right)\right]+\left[F^{\nabla}\left(e_{\imath}, X\right), \varphi\left(e_{\imath}, Y\right)\right]\right\}=0
$$

for every $X, Y \in T_{x} M$.

Proof. Let $K$ be the group of isometries of $M$ and let $k$ be its Lie algebra of Killing vector fields on $M$. Since $M$ has irreducible isotropy representation, we can fix a $K$-invariant inner product on $k$ which induces the $K$-invariant Riemannian metric of $M$. By [B-L, (10.4) Lemma], for each $V \in k$

$$
\mathcal{S}_{0}^{\nabla}\left(i_{V} \varphi\right)(X)=-\sum_{\imath=1}^{n}\left\{\left[F^{\nabla}\left(e_{\imath}, V\right), \varphi\left(e_{\imath}, X\right)\right]+\left[F^{\nabla}\left(e_{\imath}, X\right), \varphi\left(e_{\imath}, V\right)\right]\right\} .
$$

Hence $\operatorname{tr}_{k} Q_{\varphi}=0$. Since $\nabla$ is weakly stable, we have $\mathfrak{I}^{\nabla}\left(i_{V} \varphi, i_{V} \varphi\right)=0$ for all $V \in k$. For any $B \in \Omega^{1}\left(g_{E}\right)$,

$$
0 \leqq \mathfrak{T}^{\nabla}\left(i_{V} \varphi+t B, i_{V} \varphi+t B\right)=2 t \mathfrak{T}^{\nabla}\left(i_{V} \varphi, B\right)+t^{2} \mathfrak{T}^{\nabla}(B, B),
$$

hence $\mathfrak{T}^{\nabla}\left(i_{V} \varphi, B\right)=0$. Thus $\mathcal{S}_{0}^{\nabla}\left(i_{V} \varphi\right)=0$ for all $V \in k$.

q.e.d.

Consider $\Phi: M \rightarrow S^{N-1}\left(\sqrt{n / \lambda_{1}}\right) \subset \boldsymbol{R}^{N}$ be the first standard minimal immersion of $M$ (cf. [K-O-T]). Since $M$ is an Einstein manifold and $\Phi$ is a minimal immersion onto a sphere of radius $\sqrt{n / \lambda_{1}}$, if $\varphi=F^{\nabla}$, then (5.1) becomes

$$
\begin{aligned}
\mathcal{S}^{\nabla}\left(i_{U} \varphi\right)(X)= & {\left[\varphi \circ\left\{\left(\lambda_{1}-2 c\right) I+2 \mathcal{R}\right\}\right](U, X) } \\
& -2 \sum_{i, j=1}^{n}\left\langle B\left(e_{i}, e_{j}\right), u\right\rangle\left(\nabla_{e_{j}} \varphi\right)\left(e_{\imath}, X\right),
\end{aligned}
$$

where $c$ and $\lambda_{1}$ denote the Einstein constant of $M$ and the first eigenvalue of the Laplace-Beltrami operator of $M$ acting on functions, respectively.

Assume that $M$ is a compact irreducible symmetric space. Let

$$
\wedge^{2} T_{x} M=h_{0}+h_{1}+\cdots+h_{p}
$$

be the orthogonal decomposition into eigenspaces of $R$, where $h_{0}$ is the eigenspace with eigenvalue 0 and $h_{s}$ is the eigenspare with eigenvalue $\mu_{s}>0$. We 
decompose $\varphi=\varphi_{0}+\varphi_{1}+\cdots+\varphi_{p}$ along (5.5). Note that $\nabla \varphi=0$ if and only if $\nabla \varphi_{s}=0$ for each $s=0, \cdots, p$. Assume that $\nabla \varphi=0$. If $\nabla$ is weakly stable YangMills field, then by (5.3) we have

$$
\mathcal{S}^{\nabla}\left(i_{V} \varphi_{s}\right)=\left(\lambda_{1}-2 c+2 \mu_{s}\right)\left(i_{V} \varphi_{s}\right) \quad \text { for each } s=0, \cdots, p .
$$

\section{Remarks on Yang-Mills fields over compact symmetric spaces.}

First we remark on the stability of the canonical connections over compact globally Riemannian symmetric spaces. Laquer [La] determined the indices and nullities of the canonical connection on the standard principal bundle of each simply connected compact irreducible symmetric spaces. We denote by $i(\nabla)$ and $n(\nabla)$ the index and nullity of a Yang-Mills connection $\nabla$ (cf. [B-L] for their definitions).

THEOREM 6.1 ([La]). Let $M=K / H$ be a simply connected compact irreducible symmetric space associated with a symmetric pair $(K, H)$ and let $\nabla$ the canonical connection of the principal bundle $K \rightarrow K / H$.

(1) If $M$ is a group manifold, then $i(\nabla)=1$ and $n(\nabla)=0$.

(2) If $M=S^{n}(n \geqq 5), P_{2}($ Cay $), E_{6} / F_{4}$, then $i(\nabla)=n+1,26,54$ and $n(\nabla)=0$, respectively.

(3) If $M=P_{m}(H)(m \geqq 1)$, then $i(\nabla)=0, n(\nabla)=10(m=1)$ or $m(2 m+3)(m \geqq 2)$.

(4) If $M$ is otherwise, then $i(\nabla)=n(\nabla)=0$.

We should note that the values $i(\nabla)$ for $M=S^{n}(n \geqq 5), P_{2}$ (Cay), $E_{6} / F_{4}$ and $n(\nabla)$ for $M=P_{m}(H)(m \geqq 2)$ are equal to the dimension of the first eigenspace of the Laplace-Beltrami operator of $M$ acting on functions, and $n(\nabla)$ for $M=P_{1}(H)$ $=S^{4}$ is equal to its twice. It is known that, in the cases of $M=S^{n}, P_{m}(H)$, $P_{2}$ (Cay), the space of all gradient vector fields for the first eigenfunctions on $M$ coincides with the space of all proper infinitesimal conformal transformations or projective transformations on $M$.

We observe the case when $M$ is a non-simply connected, compact irreducible symmetric space. From [La] we see that if $M$ is a group manifold, then $i(\nabla)$ $=1, n(\nabla)=0$. Suppose that $M$ is not a group manifold. We easily check that if the canonical connection of the universal covering $\tilde{M}$ of $M$ has $i(\nabla)=n(\nabla)=0$, then the canonical connection of $M$ also has $i(\nabla)=n(\nabla)=0$. When $\tilde{M}=S^{n}$, by virtue of $[\mathrm{B}-\mathrm{L},(9.1)$ Theorem], we have $i(\nabla)=n(\nabla)=0$. From the theory of symmetric spaces (cf. $[\mathrm{He}]$ ) we know that if $\tilde{M}=P_{n}(H)$ or $P_{2}($ Cay), then $\tilde{M}=M$, and if $\tilde{M}=E_{6} / F_{4}$, then $M=E_{6} / F_{4} \cdot Z_{3}$. We show that the canonical connection of $M=E_{6} / F_{4} \cdot Z_{3}$ has $i(\nabla)=n(\nabla)=0$. From Theorem 6.1 we see $n(\nabla)=0$. First we recall the realization of $E_{6} / F_{4}$ and $E_{6} / F_{4} \cdot Z_{3}$ (cf. [Yo]). Consider the Jordan algebra $\mathfrak{I}=\left\{u \in M\left(3\right.\right.$, Cay); $\left.u^{*}=u\right\}$ of (real) dimension 27 . Let $R^{54}=C^{27}=\mathfrak{I}^{C}$ be the complexification of $\mathfrak{I}$ with a natural real inner product $\langle$,$\rangle . Let S^{53}=$ $\left\{u \in R^{54} ;\langle u, u\rangle=3\right\}$, a hypersphere of $\mathfrak{I}^{C}$. Set $\tilde{M}=\left\{u \in S^{53} ; \operatorname{det}(u)=1\right\}$ and let 
$\Phi$ denote the embedding $\tilde{M} \rightarrow S^{53} \subset \boldsymbol{R}^{54}$.

Proposition 6.2. (1) $\tilde{M}$ is isometric to a simply connected compact irreducible symmetric space $E_{6} / F_{4}$ (cf. [Yo]).

(2) The embedding $\Phi$ is the first standard minimal immersion of $\tilde{M}=E_{6} / F_{4}$ (cf. $\left[\mathrm{Oh}^{\top}\right]$ ).

Now we define a finite group $\Gamma$ acting freely and isometrically on $\boldsymbol{R}^{54}-\{0\}$ and $\tilde{M}$ by

$$
\begin{aligned}
& \Gamma=\left\{1, \sigma, \sigma^{2}\right\} \cong Z_{3}, \\
& \sigma(u)=e^{(2 / 3) \pi \sqrt{ }-1} u \quad \text { for each } u \in \boldsymbol{R}^{54} .
\end{aligned}
$$

Then the quotient $M=\tilde{M} / \Gamma$ is isometric to the symmetric space $E_{6} / F_{4} \cdot Z_{3}$.

Set $K=E_{6}, H=F_{4}$ and $N=54$. Let $R^{\nabla}$ be the curvature form of the cononical connection $\nabla$ for $(K, H)$. Then we have

$$
\stackrel{2}{\wedge} T_{x} \tilde{M}=\operatorname{so}\left(T_{x} \tilde{M}\right)=h_{0}+h_{1},
$$

where $h_{1}$ is isometric to the Lie algebra of $F_{4}$, which is the holonomy algebra of $\tilde{M}$. Since $\lambda_{1}-2 c+2 \mu_{1}<0$ by virtue of the result of [K-O-T], from (5.4) we see that

$$
\Theta=\left\{i_{U} R^{\nabla} ; U=\operatorname{grad} f_{u} \text { for some } u \in \boldsymbol{R}^{N}\right\}
$$

is an eigenspaces of $\mathcal{S}^{\nabla}$ of dimension 54 with a negative eigenvalue. From Theorem 6.1 we see $i(\nabla)=\operatorname{dim} \Theta$. In order to show that the canonical connection of $M$ has $i(\nabla)=0$, it suffices to show that if $i_{U} R^{\nabla} \in \Theta$ is invariant by $\Gamma$, then $U=0$. It follows from the following two lemmas.

Lemma 6.3. Let $V \in C^{\infty}(T M)$. If

$$
\gamma\left(\imath_{V} R^{\nabla}\right)=i_{V} R^{\nabla} \quad \text { for each } \gamma \in \Gamma,
$$

then $\gamma_{*} V=V$ for eoch $\gamma \in \Gamma$.

Proof. For any $X \in T_{x} M$,

$$
\begin{aligned}
R^{\nabla}\left(V_{x}, X\right) & =\gamma\left(i_{V} R^{\nabla}\right)(X)=\gamma\left(R^{\nabla}\left(V_{\gamma^{-1(x)}}, \gamma_{*}^{-1} X\right)\right) \\
& =R^{\nabla}\left(\gamma_{*} V_{\gamma^{-1(x)}}, X\right),
\end{aligned}
$$

hence $R^{\nabla}\left(\gamma_{*} V_{\gamma^{-1(x)}}-V_{x}, X\right)=0$. If we let the canonical decomposition $k=h+m$ at $x \in \tilde{M}$ and we use the identification $m=T_{x} M$, then $R^{\nabla}(X, Y)=-\operatorname{ad}_{m}[X, Y]$ (cf. [K-N]). Thus $\operatorname{ad}_{m}\left[\gamma_{*} V_{\gamma^{-1(x)}}-V_{x}, X\right]=0$ for each $X \in m$. Since $h=[m, m]$ and $k$ is semisimple, $\gamma_{*} V_{\gamma^{-1(x)}}-V_{x}=0$.

q.e.d.

Lemma 6.4. Let $U=\operatorname{grad} f_{u} \in C^{\infty}(T M)$ for some $u \in \boldsymbol{R}^{N}$. If $\gamma \in \Gamma-\{1\}$ and 
$\gamma_{*} U=U$, then $u=0$.

Proof. For each $x \in \tilde{M}$ and $X \in T_{x} M$,

$$
\left\langle\gamma_{*} U, X\right\rangle=\left\langle U, \gamma_{*}^{-1} X\right\rangle=\left\langle\gamma^{-1}(X), u\right\rangle=\langle X, \gamma(u)\rangle=\langle U, X\rangle=\langle X, u\rangle,
$$

hence $\langle X, \gamma(u)-u\rangle=0$. Thus $\langle x, \gamma(u)-u\rangle$ is constant in $x \in \tilde{M}$. Since $\Phi(\tilde{M})$ is not contained in any hyperplane of $\boldsymbol{R}^{N}$, we have $\gamma(u)=u$. Since $\Gamma$ acts freely on $\boldsymbol{R}^{N}-\{0\}$, we get $u=0$.

q.e.d.

Next we remark on weakly stable Yang-Mills fields over a quaternionic projective space $M=P_{m}(H)$. Generally let $M$ be a quaternionic Kähler manifold. The $S p(m) \cdot S p(1)$-structure induces the orthogonal decomposition

$$
\stackrel{2}{\wedge} T^{*} M=W_{0}+W_{1}+W_{2},
$$

where $\left(W_{0}\right)_{x},\left(W_{1}\right)_{x} \cong s p(1),\left(W_{2}\right)_{x} \cong s p(m)$ are irreducible $S p(m) \cdot S p(1)$-modules. The curvature form $F^{\nabla}=F_{0}^{\nabla}+F_{1}^{\nabla}+F_{2}^{\nabla}$ of a connection $\nabla$ on the vector bundle $E$ over $M$ splits into components $F_{\imath}^{\nabla}$ to $E n d(E) \otimes W_{\imath}$ at each point. A connection $\nabla$ with $F^{\nabla}=F_{2}^{\nabla}$ (resp. $F^{\nabla}=F_{1}^{\nabla}$ ) is called a $B_{2}$-connection (resp. $A_{1}^{\prime}$-connection) as in [Ni], or a self-dual connection (resp. an anti-self-dual connection) as in [C-S]. They are Yang-Mills connections which minimizes the Yang-Mills functional ([C-S], [Ni]).

Proposition 6.5. Let $E$ be a Riemannian vector bundle over $P_{m}(\boldsymbol{H})$. If $\nabla$ is a weakly stable Yang-Mills connection on $E$ satisfying $F_{1}^{\nabla}=0$, then $\nabla$ is a $B_{2^{-}}$ connection (self-dual).

Proof. We may suppose that $g$ is an $S p(m+1)$-invariant Riemannian metric on $P_{m}(\boldsymbol{H})=S p(m+1) / S p(m) \times S p(1)$ induced by the Killing form of the Lie algebra of $S p(m+1)$. From [K-O-T] we know

$$
\begin{aligned}
& \mathscr{R}=\mathcal{R}_{0}+\mathscr{R}_{1}+\mathscr{R}_{2}, \\
& \mathscr{R}_{0}=0, \\
& \mathscr{R}_{1}=(m / 2(m+2)) I, \\
& \mathscr{R}_{2}=(1 / 2(m+2)) I .
\end{aligned}
$$

Hence by virtue of (5.2), we get

$$
\begin{aligned}
& \operatorname{Tr}_{U} Q_{F^{\nabla}} \\
= & 2 \int_{M}\left(F^{\nabla}\{2 \mathscr{R}-(1 /(m+2)) I\}, F^{\nabla}\right) d v o l \\
= & 2\left\{-1 /(m+2) \int_{M}\left(F_{0}^{\nabla}, F_{0}^{\nabla}\right) d v o l+(m-1) /(m+2) \int_{M}\left(F_{1}^{\nabla}, F_{1}^{\nabla}\right) d v o l\right\},
\end{aligned}
$$


Proposition 6.5 follows from this equation.

q.e.d.

From the proof of Proposition 6.5, we see that if $\nabla$ satisfies the assumption, then

$$
\sum_{\imath, \jmath=1}^{n}\left\langle B\left(e_{\imath}, e_{\jmath}\right), u\right\rangle\left(\nabla_{e_{j}} F^{\nabla}\right)\left(e_{\imath}, X\right)=0,
$$

for all $u \in \boldsymbol{R}^{N}$ and all $X \in T_{x} M$. Using the properties of the second fundamental form of $\Phi$ and the curvature tensor field of $P_{m}(\boldsymbol{H})$, we can check that (6.2) implies that the restriction of $F^{\nabla}$ to every quaternionic projective line $P_{1}(\boldsymbol{H}) \subset$ $P_{m}(\boldsymbol{H})$ is always a Yang-Mills field. Hence by (5.6) and (6.1) we obtain that, for any $B_{2}$-connection $\nabla$ over $P_{m}(\boldsymbol{H})$ and any infinitesimal projective transformation $U$ on $P_{m}(\boldsymbol{H})$, we have $\mathcal{S}^{\nabla}\left(i_{U} F^{\nabla}\right)=0$. This means the existence of an infinitesimal action of the projective transformation group of $P_{m}(\boldsymbol{H})$ on the space of all $B_{2}$-connections over $P_{m}(\boldsymbol{H})$. In fact, it is known that the projective transformation group of $P_{m}(\boldsymbol{H})$ acts on the moduli space of all $B_{2}$-connections on $E$.

By (5.4), (5.6) and (6.1) we obtain that the indices $i(\nabla)$ and the nullity $n(\nabla)$ of the canonical connection of $M=S^{n}(n \geqq 5), P_{2}$ (Cay) and $E_{6} / F_{4}$ come from $\operatorname{span}_{R}\left\{i_{U} R^{\nabla} ; U \in \tilde{U}\right\}$, and the nullities for $M=P_{1}(\boldsymbol{H})=S^{4}$ and $P_{m}(\boldsymbol{H})(m \geqq 2)$ come from $\operatorname{span}_{R}\left\{i_{U} R_{1}^{\nabla}, i_{U} R_{2}^{\nabla} ; U \in \tilde{U}\right\}$ and $\operatorname{span}_{R}\left\{i_{U} R_{2}^{\nabla} ; U \in \tilde{U}\right\}$, respectively. We do not know whether each weakly stable canonical connection over a compact symmetric space minimizes the Yang-Mills functional. And it is interesting to investigate relationships of Yang-Mills fields with holonomy groups and the classification of vector bundles with Yang-Mills connections satisfying $\nabla F^{\nabla}=0$ over compact symmetric spaces. From results of [B-L, p. 211] and [K-O-T] we can find gap phenomena for Yang-Mills fields over every compact irreducible symmetric space which is not locally Hermitian symmetric. The classification of such Yang-Mills connections may also be useful to establish accurately isolation theorems for Yang-Mills fields over compact symmetric spaces.

Acknowledgement. This joint work was done while the second named author stayed at the Max-Planck-Institut für Mathematik in autumn, 1988. The authors would like to thank the Max-Planck Institut for the support and the hospitality.

\section{REFERENCES}

[B-L] J.P. Bourguignon And H.B. LAwson, Stability and isolation phenomena for Yang-Mills fields, Comm. Math. Phys. 79 (1981), 189-230.

[C-S] M.M. CAPRIA AND S.M. SALAmON, Yang-Mills fields on quaternionic spaces, Nonlinearity 1 (1988), 517-530.

[G-K-R1] K. GROve, H. KARChER AND E.A. RUH, Group actions and curvature, Invent. Math. 23 (1974), 31-48.

[G-K-R2] K. Grove, H. Karcher and E. A. RuH, Jacobi fields and Finsler metrics on compact Lie groups with an application to differentiable pinching problems, 
Math. Ann. 211 (1974), 7-21.

[He] S. Helgason, Differential Geometry, Lie groups and Symmetric Spaces, Academic Press, New York, San Francisco, London, 1978.

[Ka] S. KAwAI, A remark on the stability of Yang-Mills connections, Kodai Math. J. 9 (1986), 117-122.

[K-N] S. Kobayashi AND N. Nomizu, Foundations of Differential Geometry I, II, Wiley-Interscience, New York, 1963, 1969.

[K-O-T] S- Kobayashi, Y. Ohnita and M. Takeuchi, On instability of Yang-Mills connections, Math. Z. 193 (1986), 165-189.

[La] H. T. Laquer, Stability properties of the Yang-Mills functional near the cononical connection, Michigan. Math. J. 31 (1984), 139-159.

[Ni] T. NitTA, Vector bundles over quaternionic Kähler manifolds, Tohoku Math. J. 40 (1988), 425-440.

[Oh] Y. OHNiTA, The first standard minimal immersions of compact irreuducible symmetric spaces, Lecture Notes in Math. 1090, Springer-Verlag, Berlin, Heidelberg, New York, Tokyo, 1984, 37-49.

[Ok] T. OKaYAsu, Pinching and nonexistence of stable harmonic maps, Tohoku Math. J. 40 (1988), 213-220.

[Pal] Y.L. PAN, Pinching conditions for Yang-Mills instability of hypersurfaces, preprint, International Center for Theoretical Physics, Trieste, 1988.

[Pa2] Y.L. PAN, Stable harmonic maps from pinched manifolds, preprint, Max-PlanckInstitut für Math., Bonn, 1988.

[Ru] E. A. RuH, Curvature and differentiable structure on spheres, Comment. Math. Helv. 46 (1971), 127-136.

[Sh] C.L. SHEN, Weakly stability of Yang-Mills fields over the submanifold of the sphere, Arch. Math. 39 (1982), 78-84.

[Ta] C.H. Taubes, Stability in Yang-Mills theories, Comm. Math. Phys. 91 (1983), 235-263.

[We] S.W. WeI, On topological vanishing theorems and the stability of Yang-Mills fields, Indiana Univ. Math. J. 33 (1984), 511-529.

[Yo] I. Yokota, Simply connected compact Lie group $E_{6(-78)}$ of type $E_{6}$ and its involutive automorphisms, J. Math. Kyoto Univ. 20-3 (1980), 447-473.

\author{
Y. Ohnita \\ DePARTMENT OF MATHEMATICS \\ Tokyo Metropolitan University \\ Fukasawa, Setagaya \\ TOKYO 158, JAPAN
}

\author{
Y.L. PAN \\ Institute of Mathematics \\ FUDAN UNIVERSITY \\ ShanghaI \\ People's Republic of China
}

